\title{
Brønsted Acid-Promoted Olefin Aziridination And Formal anti-Aminohydroxylation
}

\author{
Joseph M. Mahoney, Colin R. Smith, and Jeffrey N. Johnston* \\ Department of Chemistry, Indiana University \\ 800 East Kirkwood Avenue, Bloomington, IN 47405
}

\section{Experimental Section}

Flame-dried (under vacuum) glassware was used for all reactions. All reagents and solvents were commercial grade and purified prior to use when necessary. Tetrahydrofuran (THF) and dichloromethane $\left(\mathrm{CH}_{2} \mathrm{Cl}_{2}\right)$ were dried by passage through a column of activated alumina as described by Grubbs. ${ }^{1}$ The carbamates ${ }^{2}$ were prepared as reported in literature.

Thin layer chromatography (TLC) was performed using glass-backed silica gel (250 $\mu \mathrm{m})$ plates and flash chromatography utilized 230-400 mesh silica gel from Scientific Adsorbents. UV light, and/or the use of ceric ammonium molybdate and potassium iodoplatinate solutions to visualize products.

IR spectra were recorded on a Nicolet Avatar 360 spectrophotometer and are reported in wavenumbers $\left(\mathrm{cm}^{-1}\right)$. Liquids and oils were analyzed as neat films on a $\mathrm{NaCl}$ plate (transmission), whereas solids were applied to a diamond plate (ATR). Nuclear magnetic resonance spectra (NMR) were acquired on either a Varian INOVA-400 (400 MHz), VXR-400 (400 MHz), or Varian INOVA-500 (500 MHz) instrument. Chemical shifts are measured relative to residual solvent peaks as an internal standard set to $\delta 7.26$ and $\delta 77.1\left(\mathrm{CDCl}_{3}\right)$. Mass spectra were recorded on a Kratos MS-80 spectrometer by use of chemical ionization (CI). Atlantic Microlabs, GA, performed combustion analyses.

\section{General Procedure for Acid Catalyzed Aziridination}

A solution of the Michael acceptor (1.0 equiv) in the indicated solvent $(0.3 \mathrm{M})$ was cooled to $0{ }^{\circ} \mathrm{C}$ and treated with triflic acid (1.2 equiv). Benzyl azide (1.5 equiv) was then added and the reaction was allowed to stir until complete conversion (generally 12 hours). The reaction mixture was then diluted with ethyl acetate and washed with $1 \mathrm{M}$ aq $\mathrm{NaOH}$. The organic layer was dried and concentrated to an oil that was purified by flash chromatography to provide the desired aziridine in analytically pure form.

\footnotetext{
${ }^{1}$ Pangborn, A. B.; Giardello, M.A.; Grubbs, R. H.; Rosen, R. K.; Timmers, F. J. Organometallics 1996, 15, 1518-1520.

${ }^{2}$ Kanazawa, A. M.; Denis, J.; Greene, A.E. J. Org. Chem. 1994, 59, 1238-1240.
} 


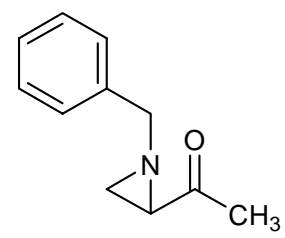

1-(1-Benzyl-aziridin-2-yl)-ethanone (2a). According to the general procedure, 3a was prepared as a colorless oil (138 mg, 79\%). $\mathrm{R}_{f}=$ 0.50 (30\% EtOAc/hexanes); IR (film) 3030, 2921, $1701 \mathrm{~cm}^{-1} ;{ }^{1} \mathrm{H}$ NMR $\left(400 \mathrm{MHz}, \mathrm{CDCl}_{3}\right) \delta$ 7.30-7.20 (m, 5H), $3.58(\mathrm{~d}, J=13.4 \mathrm{~Hz}, 1 \mathrm{H})$, 3.46 (d, $J=13.4 \mathrm{~Hz}, 1 \mathrm{H}$ ), 2.22-2.20 (m, 2H), 2.04 (s, 3H), 1.80 (dd, $J$ = 6.1, $2.4 \mathrm{~Hz}, 1 \mathrm{H}) ;{ }^{13} \mathrm{C} \mathrm{NMR}\left(100 \mathrm{MHz}, \mathrm{CDCl}_{3}\right.$ ) ppm 204.6, 138.2, 128.6, 128.1, 127.5, 64.0, 45.4, 35.0, 25.1; HRMS (EI) Exact mass calcd for $\mathrm{C}_{11} \mathrm{H}_{13} \mathrm{NO}[\mathrm{M}]^{+}, 175.0997$. Found 175.0996.

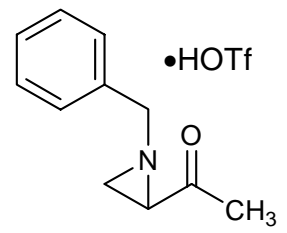

1-(1-Benzyl-aziridin-2-yl)-ethanone triflic acid salt (2a-HOTf). According to the general procedure, 3a was prepared by recrystallization (ethyl acetate/hexanes) of the crude salt as a colorless crystalline solid (748 mg, 92\%). $\mathrm{Mp}=97-100{ }^{\circ} \mathrm{C}$; IR (film) 3506, 3067, 1732, $1634 \mathrm{~cm}^{-1}$; ${ }^{1} \mathrm{H}$ NMR (400 MHz, $\mathrm{CDCl}_{3}$ ) $\delta 8.30$ (br s, $1 \mathrm{H}$ ), 7.48-7.30 (m, 5H), 4.56 (d, $J=13.2 \mathrm{~Hz}, 1 \mathrm{H}$ ), 4.44 (d, $J=13.2 \mathrm{~Hz}, 1 \mathrm{H}$ ), 4.08 (dd, $J=7.4$, $6.2 \mathrm{~Hz}, 1 \mathrm{H}$ ), 3.06 (d, $J=6.2 \mathrm{~Hz}, 1 \mathrm{H}$ ), 3.01 (d, $J=7.4 \mathrm{~Hz}, 1 \mathrm{H}$ ), 2.39 (s, 3H); ${ }^{13} \mathrm{C}$ NMR (100 MHz, $\mathrm{CDCl}_{3}$ ) ppm 197.8, 130.6, 130.5, 129.7, 129.0, 55.6, 44.4, 35.8, 29.3.

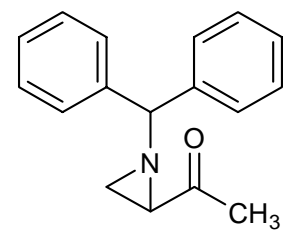

1-(1-Benzhydryl-aziridin-2-yl)-ethanone (2b). According to the general procedure $\mathbf{2 b}$ was prepared as a colorless oil (1.50 g, 88\%). $\mathrm{R}_{f}$ $=0.27$ (30\% EtOAc/hexanes); IR (film) 3061, 3027, 1703, 1493, 1453 $\mathrm{cm}^{-1}$; ${ }^{1} \mathrm{H}$ NMR (400 MHz, $\left.\mathrm{CDCl}_{3}\right) \delta$ 7.47-7.23 (m, 10H), $3.63(\mathrm{~s}, 1 \mathrm{H})$, 2.31-2.29 (m, 2H), 2.10 (s, 3H), 1.89 (d, $J=6.1 \mathrm{~Hz}, 1 \mathrm{H}) ;{ }^{13} \mathrm{C}$ NMR (100 MHz, $\mathrm{CDCl}_{3}$ ) ppm 207.1, 142.7, 128.7, 127.5, 127.2, 77.9, 45.9, 35.2, 25.1; HRMS (EI) Exact mass calcd for $\mathrm{C}_{17} \mathrm{H}_{18} \mathrm{NO}[\mathrm{M}+\mathrm{H}]^{+}$, 252.1388. Found 252.1391. Anal. Calcd for $\mathrm{C}_{17} \mathrm{H}_{17} \mathrm{NO}$ : C, 81.24; H, 6.82; N, 5.57. Found: C, 81.11; H, 6.87; N, 5.59.

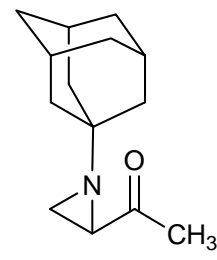

1-(1-Adamantan-1-yl-aziridin-2-yl)-ethanone (2c). According to the general procedure, $2 \mathrm{c}$ was prepared as a white solid (93\%). $\mathrm{R}_{f}=0.43(20 \%$ EtOAc/hexanes); IR (film) 2918, 1703. ${ }^{1} \mathrm{H}$ NMR (400 MHz, $\left.\mathrm{CDCl}_{3}\right) \delta$ 2.43 (dd, $J=6.8,2.8 \mathrm{~Hz}, 1 \mathrm{H},), 2.06$ (m, 3H), 2.03 (s, 3H), 2.01 (dd, $J=$ 6.8, $1.2 \mathrm{~Hz}, 1 \mathrm{H}), 1.83$ (dd, $J=2.8,1.2 \mathrm{~Hz}, 1 \mathrm{H}), 1.58(\mathrm{~m}, 12 \mathrm{H}) .{ }^{13} \mathrm{C} \mathrm{NMR}$ (100 MHz, $\mathrm{CDCl}_{3}$ ) ppm 209.5, 53.4, 40.4, 36.8, 29.6, 26.9, 24.9. HRMS (EI): Exact mass calcd for $\mathrm{C}_{14} \mathrm{H}_{21} \mathrm{NO}[\mathrm{M}]^{+}$, 219.1623. Found 219.1621. Anal. Calcd for $\mathrm{C}_{14} \mathrm{H}_{21} \mathrm{NO}: \mathrm{C}$, 71.57; H, 6.86; N, 6.39. Found: C, 71.16; H, 6.91; N, 6.27. 


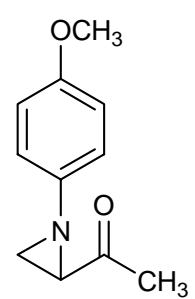

1-[1-(4-Methoxy-phenyl)-aziridin-2-yl]-ethanone (2d). According to the general procedure $2 \mathbf{d}$ was prepared as a yellow oil (30 mg, 43\%). $\mathrm{R}_{f}=0.12$ (30\% EtOAc/hexanes); IR (film) 2918, 2835, 1703, $1507 \mathrm{~cm}^{-1}$; ${ }^{1} \mathrm{H}$ NMR $\left(400 \mathrm{MHz}, \mathrm{CDCl}_{3}\right.$ ) $\delta 6.87$ (ddd, $J=8.8,3.6,2.6 \mathrm{~Hz}, 2 \mathrm{H}$ ), 6.76 (ddd, $J=8.8$, 3.6, $2.6 \mathrm{~Hz}, 2 \mathrm{H}$ ), 3.73 (s, 3H), 2.65 (dd, $J=2.8,6.8 \mathrm{~Hz}, 1 \mathrm{H}$ ), 2.52 (dd, $J=$ 2.8, $1.6 \mathrm{~Hz}, 1 \mathrm{H}), 2.27$ (dd, $J=6.8,1.6 \mathrm{~Hz}, 1 \mathrm{H}), 2.13$ (s, 3H); ${ }^{13} \mathrm{C}$ NMR (100 $\mathrm{MHz}_{\mathrm{CDCl}}$ ) ppm 206.8, 155.9, 145.9, 121.5, 114.6, 55.7, 45.6, 34.3, 25.0; HRMS (EI): Exact mass calcd for $\mathrm{C}_{11} \mathrm{H}_{13} \mathrm{NO}_{2}[\mathrm{M}]^{+}$, 191.0940. Found 191.0946. Anal. Calcd for $\mathrm{C}_{11} \mathrm{H}_{13} \mathrm{NO}_{2}$ : C, 69.09; H, 6.85; N, 7.32. Found: C, 68.81; H, 6.90; N, 7.48.

(2-Acetyl-aziridin-1-yl)-acetic acid tert-butyl ester (2e).

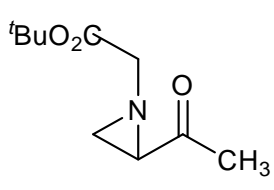

According to the general procedure $2 \mathbf{e}$ was prepared as a colorless oil (132 mg, 66\%). $\mathrm{R}_{f}=0.56$ (40\% EtOAc/hexanes); IR (film) 2980, 2927, 1740, 1704, $1368 \mathrm{~cm}^{-1} ;{ }^{1} \mathrm{H}$ NMR (400 MHz, $\left.\mathrm{CDCl}_{3}\right) \delta$ 3.19 (d, $J=16.1 \mathrm{~Hz}, 1 \mathrm{H}), 2.91$ (d, $J=16.1 \mathrm{~Hz}, 1 \mathrm{H}), 2.23$ (d, $J=3.3$ $\mathrm{Hz}, 1 \mathrm{H}), 2.08$ (s, 3H), 1.72 (d, $J=6.9 \mathrm{~Hz}, 1 \mathrm{H}), 1.46$ (s, 9H); ${ }^{13} \mathrm{C}$ NMR (100 MHz, $\mathrm{CDCl}_{3}$ ) ppm 206.9, 168.7, 81.9, 61.5, 45.4, 34.3, 28.2, 25.1; HRMS (CI) Exact mass calcd for $\mathrm{C}_{10} \mathrm{H}_{7} \mathrm{NO}_{3}[\mathrm{M}+\mathrm{H}]^{+}, 200.1286$. Found 200.1284 .

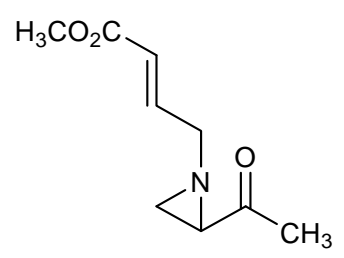

4-(2-Acetyl-aziridin-1-yl)-but-2-enoic acid methyl ester (2f). According to the general procedure $2 \mathbf{f}$ was prepared as a colorless oil (152 mg, 75\%). $\mathrm{R}_{f}=0.10$ (30\% EtOAc/hexanes); IR (film) 3003, 2948, 2834, 1728, 1701, $1445 \mathrm{~cm}^{-1}$; ${ }^{1} \mathrm{H}$ NMR $(400 \mathrm{MHz}$, $\left.\mathrm{CDCl}_{3}\right) \delta 6.89(\mathrm{dt}, J=15.5,4.9 \mathrm{~Hz}, 1 \mathrm{H}), 6.00(\mathrm{dt}, J=15.5,1.8 \mathrm{~Hz}$, 1H), 3.67 (s, 3H), 3.06 (ddd, $J=16.5,4.9,1.9 \mathrm{~Hz}, 2 \mathrm{H}$ ), 2.12 (d, $J=3.2 \mathrm{~Hz}, 1 \mathrm{H}$ ), 2.07 (dd, $J=7.1,3.2 \mathrm{~Hz}, 1 \mathrm{H}), 2.00$ (s, 3H), 1.63 (d, $J=7.1 \mathrm{~Hz}, 1 \mathrm{H}) ;{ }^{13} \mathrm{C}$ NMR (100 MHz, $\left.\mathrm{CDCl}_{3}\right)$ ppm 206.4, 166.5, 144.0, 121.9, 60.3, 51.6, 45.1, 34.6, 25.0; HRMS (EI) Exact mass calcd for $\mathrm{C}_{9} \mathrm{H}_{13} \mathrm{NO}_{3}[\mathrm{M}+\mathrm{H}]^{+}$, 184.0971. Found 184.0971.

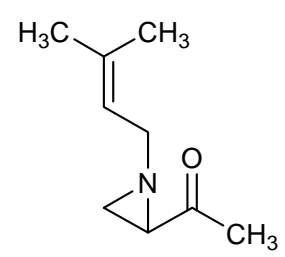

1-[1-(3-Methyl-but-2-enyl)-aziridin-2-yl]-ethanone (2g). According to the general procedure $\mathbf{2 f}$ was prepared as a colorless oil (105 mg, 68\%). $\mathrm{R}_{f}=0.28$ (30\% EtOAc/hexanes); IR (film) 2965, 2921, 2850, 1701, $1353 \mathrm{~cm}^{-1} ;{ }^{1} \mathrm{H}$ NMR (400 MHz, $\left.\mathrm{CDCl}_{3}\right) \delta 5.26(\mathrm{t}, J=5.8 \mathrm{~Hz}$, 1H), 2.95 (dd, $J=13.2,5.8 \mathrm{~Hz}, 1 \mathrm{H}$ ), 2.91 (dd, $J=13.2,5.8 \mathrm{~Hz}, 1 \mathrm{H}$ ), 2.08-2.06 (m, 2H), 2.01 (s, 3H), 1.72 (s, 3H), 1.65 (d, J = 7.1 Hz, 1H), 1.60 (s, 3H); ); ${ }^{13} \mathrm{C}$ 
NMR (100 MHz, $\mathrm{CDCl}_{3}$ ) ppm 208.2, 136.1, 120.7, 58.0, 45.1, 34.7, 25.9, 25.0, 18.3; HRMS (EI) Exact mass calcd for $\mathrm{C}_{9} \mathrm{H}_{15} \mathrm{NO}[\mathrm{M}]^{+}, 153.1154$. Found 153.1153.

\section{General Procedure for Acid Catalyzed anti-Aminohydroxylation}

To a vial, the Michael acceptor (1.0 equiv) was added with solvent to generate a $0.3 \mathrm{M}$ solution. The solution was cooled to $-20{ }^{\circ} \mathrm{C}$ and benzyl azide (2.0 equiv) was added. Triflic acid (2.0 equiv) was then added, and the reaction mixture was stirred until complete conversion (generally 12-48 hours). The reaction was diluted with triethyl amine ( 5 equiv) and concentrated to an oil that was purified by flash chromatography to give the analytically pure amine.

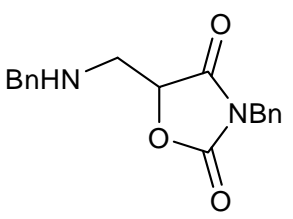

\section{3-Benzyl-5-(benzylamino-methyl)-oxazolidine-2,4-dione}

(3a).

According to the general procedure 3a was prepared as a colorless oil (143 mg, 84\%). $\mathrm{R}_{f}=0.13$ (30\% EtOAc/hexanes); IR (film) 3350, 3031, 2933, 2840, 1815, 1739, 1439 $\mathrm{cm}^{-1}$; ${ }^{1} \mathrm{H}$ NMR $(400 \mathrm{MHz}$, $\left.\mathrm{CDCl}_{3}\right) \delta$ 7.42-7.40 (m, 2H), 7.30-7.25 (m, 6H), 7.19-7.17 (m, 2H), 4.84 (t, $J=3.3 \mathrm{~Hz}$, 1H), 4.72 (d, $J=14.3 \mathrm{~Hz}, 1 \mathrm{H}$ ), 4.67 (d, $J=14.3 \mathrm{~Hz}, 1 \mathrm{H}$ ), 3.77 (s, 2H), 3.21 (dd, $J=13.8$, 3,3 Hz, 1H), 3.11 (dd, $J=13.8,3.3 \mathrm{~Hz}, 1 \mathrm{H}$ ); ${ }^{13} \mathrm{C}$ NMR (100 MHz, $\mathrm{CDCl}_{3}$ ) ppm 172.1, 155.6, 139.4, 134.7, 128.9, 128.6, 128.3, 128.1, 127.3, 80.2, 60.5, 54.0, 47.9, 43.8, 21.2, 14.3; HRMS (EI) Exact mass calcd for $\mathrm{C}_{18} \mathrm{H}_{19} \mathrm{~N}_{2} \mathrm{O}_{3}[\mathrm{M}+\mathrm{H}]^{+}$, 311.1395. Found 311.1392. Anal. Calcd for $\mathrm{C}_{18} \mathrm{H}_{18} \mathrm{~N}_{2} \mathrm{O}_{3}$ : C, 69.66; H, 5.85; N, 9.03. Found: C, 69.81; H, 5.89; N, 8.91 .

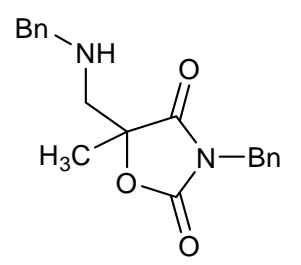

3-Benzyl-5-(benzylamino-methyl)-5-methyl-oxazolidine-2,4dione (3b). According to the general procedure $\mathbf{3 b}$ was prepared as a colorless oil (123 mg, 88\%). $\mathrm{R}_{f}=0.63$ (50\% EtOAc/hexanes); IR (film) 3400, 3046, 2937, 2844, 1804, 1733, $1411 \mathrm{~cm}^{-1}$; ${ }^{1} \mathrm{H}$ NMR (400 MHz, $\left.\mathrm{CDCl}_{3}\right) \delta$ 7.36-7.31 (m, 2H), 7.30-7.24 (m, 3H), 7.17 (d, $J=7.6 \mathrm{~Hz}, 1 \mathrm{H}$ ), 7.15 (d, $J=7.3 \mathrm{~Hz}, 1 \mathrm{H}), 6.73$ (t, $J=7.2 \mathrm{~Hz}, 1 \mathrm{H}), 6.47$ (d, $J=8.0 \mathrm{~Hz}$, 2H), 4.60 (d, $J=14.6 \mathrm{~Hz}, 1 \mathrm{H}), 4.54$ (d, $J=14.6 \mathrm{~Hz}, 1 \mathrm{H}$ ), 3.47 (bs, 1H), 3.41 (t, $J=6.7$ $\mathrm{Hz}, 2 \mathrm{H}$ ), 2.21 (dq, $J=14.6,7.3 \mathrm{~Hz}, 2 \mathrm{H}), 1.58$ (s, 3H); ${ }^{13} \mathrm{C} \mathrm{NMR}\left(100 \mathrm{MHz}, \mathrm{CDCl}_{3}\right.$ ) ppm 175.4, 154.7, 147.5, 134.9, 129.5, 129.1, 128.7, 128.5, 118.3, 113.2, 85.2, 43.9, 38.6, 36.3, 23.3; HRMS (EI) Exact mass calcd for $\mathrm{C}_{19} \mathrm{H}_{20} \mathrm{~N}_{2} \mathrm{O}_{3}[\mathrm{M}]^{+}$, 324.1474. Found 324.1463. Anal. Calcd for $\mathrm{C}_{19} \mathrm{H}_{20} \mathrm{~N}_{2} \mathrm{O}_{3}$ : C, 70.35; H, 6.21; N, 8.64. Found: C, 70.54; H, 6.22; N, 8.62 . 


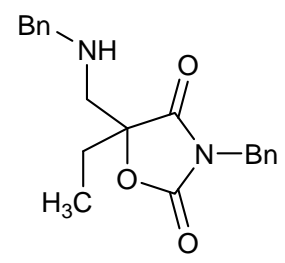

3-Benzyl-5-(benzylamino-methyl)-5-ethyl-oxazolidine-2,4-dione (3c). According to the general procedure 3c was prepared as a colorless oil (35 mg, 61\%). $\mathrm{R}_{f}=0.14$ (20\% EtOAc/hexanes); IR (film) 3403, 3033, 2927, 1810, 1733, $1603 \mathrm{~cm}^{-1} ;{ }^{1} \mathrm{H}$ NMR (400 MHz, $\mathrm{CDCl}_{3}$ ) $\delta$ 7.37-7.32 (m, 2H), 7.28-7.24 (m, 3H), 7.18-7.13 (m, 2H), 6.75-6.71

(m, 1H), 6.50-6.45 (m, 2H), 4.56 (AB q, $J=14.4 \mathrm{~Hz}, 2 \mathrm{H}), 3.50$ (br s, 1H), 3.14 (t, $J=6.6$ $\mathrm{Hz}, 2 \mathrm{H}), 2.28-2.13$ (m, 2H) 1.98-1.89 (m, 2H), 0.82 (t, $J=7.6 \mathrm{~Hz}, 3 \mathrm{H}) ;{ }^{13} \mathrm{C}$ NMR (100 $\mathrm{MHz}_{\mathrm{CDCl}}$ ) ppm 175.0, 155.2, 147.5, 134.9, 129.5, 129.0, 128.8, 128.5, 118.3, 113.2, 88.3, 43.9, 38.5, 35.3, 29.9; HRMS (EI): Exact mass calcd for $\mathrm{C}_{20} \mathrm{H}_{22} \mathrm{~N}_{2} \mathrm{O}_{3}[\mathrm{M}]^{+}$, 338.1630. Found 338.1625. Anal. Calcd for $\mathrm{C}_{20} \mathrm{H}_{22} \mathrm{~N}_{2} \mathrm{O}_{3}$ : C, 70.99; H, 6.55; N, 8.28. Found: C, 70.85; H, 6.49; N, 8.20.

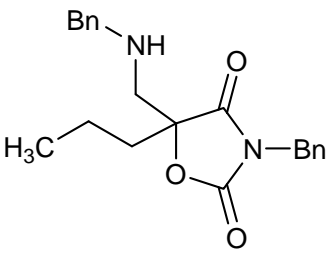

3-Benzyl-5-(benzylamino-methyl)-5-propyl-oxazolidine-2,4dione (3d). According to the general procedure 3d was prepared as a colorless oil (35 mg, 60\%). $\mathrm{R}_{f}=0.13$ (10\% EtOAc/hexanes); IR (film) 3394, 2962, 2924, 1810, $1734 \mathrm{~cm}^{-1}$; ${ }^{1} \mathrm{H}$ NMR (400 MHz, $\left.\mathrm{CDCl}_{3}\right) \delta 7.42-7.22(\mathrm{~m}, 5 \mathrm{H}), 7.17-7.11(\mathrm{~m}, 2 \mathrm{H}), 6.72(\mathrm{tt}, J=7.6$, $1.2 \mathrm{~Hz}, 1 \mathrm{H}), 6.46$ (m, 2H), 4.55 (AB q, $J=14.4 \mathrm{~Hz}, 2 \mathrm{H}), 3.12$ (t, $J=7.2 \mathrm{~Hz}, 2 \mathrm{H}), 2.18$ (m, 2H), $1.84(\mathrm{~m}, 2 \mathrm{H}), 1.32(\mathrm{~m}, 1 \mathrm{H}), 1.16(\mathrm{~m}, 1 \mathrm{H}), 0.86(\mathrm{t}, J=7.2 \mathrm{~Hz}, 3 \mathrm{H}) ;{ }^{13} \mathrm{C}$ NMR (100 MHz, $\left.\mathrm{CDCl}_{3}\right)$ ppm 175.0, 155.1, 147.5, 134.9, 129.5, 129.0, 128.8, 128.5, 118.3, 113.2, 88.0, 43.9, 38.7, 38.5, 35.5, 16.1, 13.9; HRMS (CI): Exact mass calcd for $\mathrm{C}_{21} \mathrm{H}_{24} \mathrm{~N}_{2} \mathrm{O}_{3}[\mathrm{M}]^{+}$, 352.1783. Found 352.1787. Anal. Calcd for $\mathrm{C}_{21} \mathrm{H}_{24} \mathrm{~N}_{2} \mathrm{O}_{3}: \mathrm{C}, 71.57 ; \mathrm{H}$, 6.86; N, 7.95. Found: C, 71.59; H, 6.86; N, 7.90.

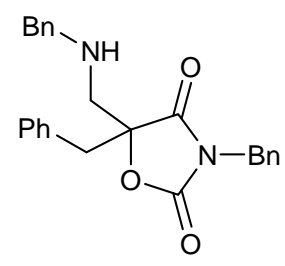

3,5-Dibenzyl-5-(benzylamino-methyl)-oxazolidine-2,4-dione (3e). Compound 3e was prepared according to the general procedure except it was allowed to react in a sealed tube and heated at $50{ }^{\circ} \mathrm{C}$ for 7 days. Colorless oil (27 mg, 46\%). $\mathrm{R}_{f}=0.15$ (20\% EtOAc/hexanes); IR (film) 3403, 3031, 2924, 1815, 1734, $1603 \mathrm{~cm}^{-1} ;{ }^{1} \mathrm{H}$ NMR (400 MHz, $\mathrm{CDCl}_{3}$ ) $\delta$ 7.20-7.11 (m, 10H), 6.93-6.89 (m, 2H), 6.77-6.71 (m, 1H), 6.53-6.48 (m, 2H), 4.24 (AB q, $J=14.4 \mathrm{~Hz}, 2 \mathrm{H}$ ), 3.50 (br s, $1 \mathrm{H}), 3.23$ (m, 2H), 3.17 (s, 2H), 2.44-2.36 (m, 1H), 2.322.24 (m, 1H); ${ }^{13} \mathrm{C}$ NMR (100 MHz, $\mathrm{CDCl}_{3}$ ) ppm 174.2, 154.6, 147.5, 134.4, 132.3, 130.4, 129.5, 128.9, 128.8, 128.1, 128.0, 118.4, 113.2, 54.2, 43.6, 42.5, 38.6, 35.7; HRMS (CI): Exact mass calcd for $\mathrm{C}_{25} \mathrm{H}_{24} \mathrm{~N}_{2} \mathrm{O}_{3}[\mathrm{M}]^{+}$, 400.1787. Found 400.1794. Anal. Calcd for $\mathrm{C}_{25} \mathrm{H}_{24} \mathrm{~N}_{2} \mathrm{O}_{3}$ : C, 74.98; H, 6.04; N, 7.00. Found: C, 74.96; H, 6.13; N, 6.85. 


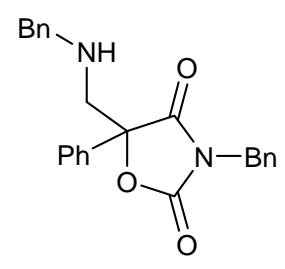

3-Benzyl-5-(benzylamino-methyl)-5-phenyl-oxazolidine-2,4-dione (3f). According to the General Procedure, 3f was prepared as a colorless oil (28 mg, 86\%). $\mathrm{R}_{f}=0.46$ (20\% EtOAc/hexanes); IR (film) 3034, 2955, 1742, 1674, $1615 \mathrm{~cm}^{-1} ;{ }^{1} \mathrm{H}$ NMR (400 MHz, $\mathrm{CDCl}_{3}$ ) $\delta$ 7.64-7.58 (m, 2H), 7.45-7.38 (m, 3H), 7.34-7.24 (m, 5H), 7.18-7.12 (m, 2H), 6.73 (t, $J=7.4 \mathrm{~Hz}, 1 \mathrm{H}), 6.45$ (d, $J=8.8 \mathrm{~Hz}, 2 \mathrm{H}), 4.60$ (dd, $J=14.8,4.0 \mathrm{~Hz}$, 2H), 3.48 (br s, $1 \mathrm{H}$ ), 3.18 (m, 2H), 2.50 (m, 2H); ${ }^{13} \mathrm{C} \mathrm{NMR} \mathrm{(100} \mathrm{MHz,} \mathrm{CDCl}_{3}$ ) ppm 173.5, 154.6, 147.4, 137.8, 134.6, 129.5, 129.3, 129.2, 129.1, 128.61, 128.56, 124.7, 118.3, 113.3, 87.4, 44.2, 38.8, 38.4; HRMS (CI): Exact mass calcd for $\mathrm{C}_{24} \mathrm{H}_{22} \mathrm{~N}_{2} \mathrm{O}_{3}[\mathrm{M}]^{+}$ 386.1630. Found 386.1629.

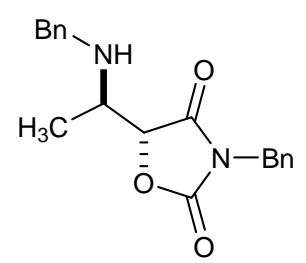

3-Benzyl-5-(1-benzylamino-ethyl)-oxazolidine-2,4-dione

(3g). According to the general procedure $\mathbf{3 g}$ was prepared as a colorless oil (131 mg, 94\%). $\quad \mathrm{R}_{f}=0.30$ (30\% EtOAc/hexanes). IR (film) 3300, 3063, 2933, 2850, 1814, $1733 \mathrm{~cm}^{-1}$; ${ }^{1} \mathrm{H} \mathrm{NMR} \mathrm{(400} \mathrm{MHz,} \mathrm{CDCl}_{3}$ ) $\delta$ 7.41-7.23 (m, 10H), 4.82 (d, $J=3.1 \mathrm{~Hz}, 1 \mathrm{H}), 4.72-4.64$ (m, 3H), 3.84 (d, $J=13.2 \mathrm{~Hz}, 1 \mathrm{H}), 3.78(\mathrm{~d}, J=13.2 \mathrm{~Hz}, 1 \mathrm{H}), 3.30-3.23$ (m, 1H), 1.43 (bs, $1 \mathrm{H}), 1.14$ (d, $J=6.8 \mathrm{~Hz}, 3 \mathrm{H}) ;{ }^{13} \mathrm{C} \mathrm{NMR}\left(100 \mathrm{MHz}, \mathrm{CDCl}_{3}\right) \mathrm{ppm}$ 171.7, 155.6, 139.6, 134.7, 129.1, 129.0, 128.9, 128.7, 128.6, 128.4, 128.1, 127.3, 82.0, 53.5, 51.8, 43.7, 15.1; HRMS (EI): Exact mass calcd for $\mathrm{C}_{19} \mathrm{H}_{21} \mathrm{~N}_{2} \mathrm{O}_{3}[\mathrm{M}+\mathrm{H}]^{+}$, 325.1553. Found 325.156. Anal. Calcd for $\mathrm{C}_{18} \mathrm{H}_{18} \mathrm{~N}_{2} \mathrm{O}_{3}$ : C, 70.35; H, 6.21; N, 8.64. Found: C, 70.54; H, 6.22; N, 8.51. Relative configuration was assigned by X-ray analysis of the $\beta$-dibenzylamino derivative.

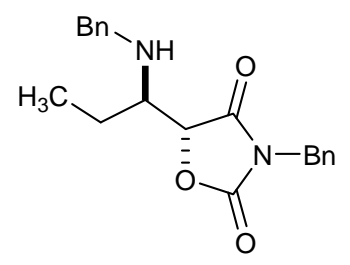

3-Benzyl-5-(1-benzylamino-propyl)-oxazolidine-2,4-dione (3h). According to the general procedure $3 \mathbf{h}$ was prepared as a colorless oil (125 mg, 82\%). Major diastereomer. $\mathrm{R}_{f}=0.20 \quad(30 \%$ EtOAc/hexanes). IR (film) 2920, 2849, 1735, $1646 \mathrm{~cm}^{-1} .{ }^{1} \mathrm{H}$ NMR $\left(400 \mathrm{MHz}, \mathrm{CDCl}_{3}\right) \delta 7.44-7.18(\mathrm{~m}, 10 \mathrm{H}), 4.84$ (dd, $\left.J=2.8 \mathrm{~Hz}, 1 \mathrm{H}\right)$, 4.67 (d, $J=7.2 \mathrm{~Hz}, 2 \mathrm{H}$ ), 3.80 (d, $J=4.0 \mathrm{~Hz}, 2 \mathrm{H}), 3.12$ (m, 1H), 1.60 (m, 2H), 0.97 (t, $J=$ $7.6 \mathrm{~Hz}, 3 \mathrm{H}) .{ }^{13} \mathrm{C} \mathrm{NMR}\left(100 \mathrm{MHz}, \mathrm{CDCl}_{3}\right.$ ) ppm 172.0, 155.8, 139.9, 134.9, 129.0, 128.8, 128.7, 128.5, 128.3, 127.4, 81.7, 60.0, 53.2, 43.8, 23.3, 11.0. HRMS (EI): Exact mass calcd for $\mathrm{C}_{20} \mathrm{H}_{22} \mathrm{~N}_{2} \mathrm{O}_{3}[\mathrm{M}-\mathrm{H}]^{+}$, 337.1552. Found 337.1542. 


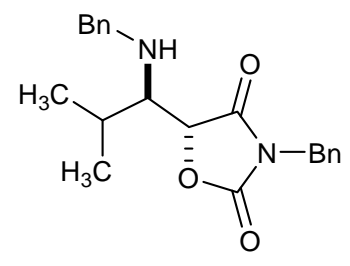

3-Benzyl-5-(1-benzylamino-2-methyl-propyl)-oxazolidine-2,4dione (3i). According to the general procedure $3 \mathbf{i}$ was prepared as a colorless oil (26 mg, 61\%). Major diastereomer; $\mathrm{R}_{f}=0.20 \quad(30 \%$

EtOAc/hexanes). IR (film) 3430, 2923, 2852, 1735, $1656 \mathrm{~cm}^{-1} .{ }^{1} \mathrm{H}$ NMR (400 MHz, $\left.\mathrm{CDCl}_{3}\right) \delta 7.38(\mathrm{~m}, 2 \mathrm{H})$ 7.29-7.21 (m, 6H), 7.13 (m, 2H), 4.95 (d, $J=2.8 \mathrm{~Hz}, 1 \mathrm{H}), 4.68$ (dd, $J=14.4,10.8 \mathrm{~Hz}, 2 \mathrm{H}), 3.78$ (dd, $J=14.4$, $10.8 \mathrm{~Hz}, 2 \mathrm{H}$ ), 2.78 (dd, $J=8.8,3.2 \mathrm{~Hz}, 1 \mathrm{H}), 2.00$ (m, 1H), 1.05 (d, $J=6.4 \mathrm{~Hz}, 3 \mathrm{H}$ ), $1.01(\mathrm{~d}, J=6.4 \mathrm{~Hz}, 3 \mathrm{H}) .{ }^{13} \mathrm{C} \mathrm{NMR}\left(100 \mathrm{MHz}, \mathrm{CDCl}_{3}\right) \mathrm{ppm}$ 172.0, 156.1, 139.9, 134.9, 128.9, 128.7, 128.6, 128.5, 128.3, 127.4, 81.8, 65.2, 55.3, 43.8, 29.9, 20.6, 20.1. HRMS (EI) Exact mass calcd for $\mathrm{C}_{21} \mathrm{H}_{24} \mathrm{~N}_{2} \mathrm{O}_{3}[\mathrm{M}+\mathrm{H}]^{+}$, 351.1709. Found 351.1710. Anal. Calcd for $\mathrm{C}_{21} \mathrm{H}_{24} \mathrm{~N}_{2} \mathrm{O}_{3}$ : C, 71.57; H, 6.86; N, 7.95. Found: C, 71.16; H, 6.91; N, 7.70.

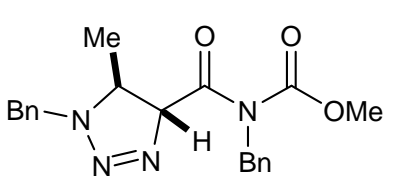

Benzyl-(1-benzyl-5-methyl-[1,2,3]triazolidine-4-carbonyl)carbamic acid methyl ester (4). Imide $1 \mathrm{~g}$ (160 mg, 683 mol) was stirred in neat benzyl azide (10 equivalents) at ambient temperature in the dark for 1 week (70\% conversion by ${ }^{1} \mathrm{H}$ NMR). Concentration of the reaction mixture and chromatography of the residue $\left(\mathrm{SiO}_{2}\right.$, $10 \%$ ethyl acetate in hexanes) furnished the triazoline as a colorless oil (138 mg, 79\%). $\mathrm{R}_{f}$ $=0.13$ (20\% EtOAc/hexanes); IR (neat) $\left(\mathrm{cm}^{-1}\right)$ : 3031, 2957, 2094, 1746, 1695; ${ }^{1} \mathrm{H} \mathrm{NMR}$ (400 MHz, $\left.\mathrm{CDCl}_{3}\right) \delta 7.38-7.20(\mathrm{~m}, 10 \mathrm{H}), 5.66(\mathrm{~d}, J=11.2 \mathrm{~Hz}, 1 \mathrm{H}), 4.93$ (dd, $J=13.2$, $9.2 \mathrm{~Hz}, 2 \mathrm{H}), 4.92$ (d, $J=14.8 \mathrm{~Hz}, 1 \mathrm{H}), 4.63$ (d, $J=14.8 \mathrm{~Hz}, 1 \mathrm{H}), 3.83$ (s, 3H), 3.72 (m, 1H), 1.16 (d, $J=6.0 \mathrm{~Hz}, 3 \mathrm{H}) ;{ }^{13} \mathrm{C}$ NMR (100 MHz, $\mathrm{CDCl}_{3}$ ) ppm 170.2, 155.1, 137.2, 135.9, 132.6, 128.8, 128.6, 128.3, 128.0, 127.7, 127.6, 86.3, 54.8, 54.2, 52.4, 48.3, 17.2 . HRMS (ES) Exact mass calcd for $\mathrm{C}_{20} \mathrm{H}_{22} \mathrm{NaN}_{4} \mathrm{O}_{3}[\mathrm{M}+\mathrm{Na}]^{+}$, 367.1765. Found 367.1764. 